\title{
Game-Theory-Based Approach for Energy Routing in a Smart Grid Network
}

\author{
June S. Hong' and Mihui Kim² \\ ${ }^{1}$ Department of Management Information Systems, Kyonggi University, 154-42 Gwanggyosan-ro, Yeongtong-gu, Suwon-si, \\ Gyeonggi-do 443-760, Republic of Korea \\ ${ }^{2}$ Department of Computer \& Web Information Engineering, Computer System Institute, Hankyong National University, \\ 327 Jungang-ro, Anseong-si, Gyeonggi-do 456-749, Republic of Korea
}

Correspondence should be addressed to Mihui Kim; mhkim@hknu.ac.kr

Received 7 October 2015; Accepted 8 December 2015

Academic Editor: Tzonelih Hwang

Copyright @ 2016 J. S. Hong and M. Kim. This is an open access article distributed under the Creative Commons Attribution License, which permits unrestricted use, distribution, and reproduction in any medium, provided the original work is properly cited.

Small power plants and buildings with renewable power generation capability have recently been added to traditional central power plants. Through these facilities, prosumers appear to have a concurrent role in both energy production and consumption. Based on bidirectional power transfers by large numbers of prosumers, a smart microgrid has become an important factor in efficiently controlling the microgrids used in power markets and in conducting effective power trades among grids. In this paper, we present an approach utilizing the game theory for effective and efficient energy routing, which is a novel and challenging procedure for a smart microgrid network. First, we propose strategies for choosing the desired transaction price for both electricity surpluses and shortages to maximize profits through energy transactions. An optimization scheme is utilized to search for an energy route with minimum cost using the solving method used in a traditional transportation problem by treating the sale and purchase quantities as transportation supply and demand, respectively. To evaluate the effect of the proposed decision strategies, we simulated our mechanism, and the results proved that our mechanism yields results pursued by each strategy. Our proposed strategies will contribute to spreading a smart microgrid for enhancing the utilization of microgrids.

\section{Introduction}

In a traditional energy market, few suppliers in large central power plants supply power to a great number of homes and businesses. However, relatively small-scale, self-contained, medium-/low-voltage electric power systems (EPSs) housing various distributed energy resources (DERs), that is, solar panels or wind turbines, have come into wide use. Prosumers thus appear to have a role in both energy production and consumption concurrently. A smart microgrid is important in efficiently controlling the microgrids in the power markets based on bidirectional power transfers by large numbers of prosumers, and in effectively performing power trades between these grids. A smart microgrid is defined as a localized grouping of electricity generation (i.e., solar panels or wind turbines) sources, energy storage facilities, and loads and may include distributed energy resources (DERs) and an intelligent distribution system with energy flow management carried out by intelligent electronic devices balancing both the load and source [1], as shown in Figure 1. Thus, energy routing in a smart microgrid is being magnified as a novel but challenging procedure in smart microgrid networks, and the efficiency, survivability, reliability, flexibility, and availability are being highlighted.

In particular, researchers have focused on the effective and efficient energy routing in smart microgrid networks to fully utilize the distributed energy resources and decrease the cost of energy transmissions. Similar to traditional routing protocols [2], energy routers exchange the energy information of each home in the data network and try to find the most effective transaction and efficient transmission path in the energy network for energy sharing among homes. Differing from traditional routing protocols, the routing mechanism has an energy plane but no data plane. After the energy routers find the most effective transaction and efficient transmission path in the control plane of the data 


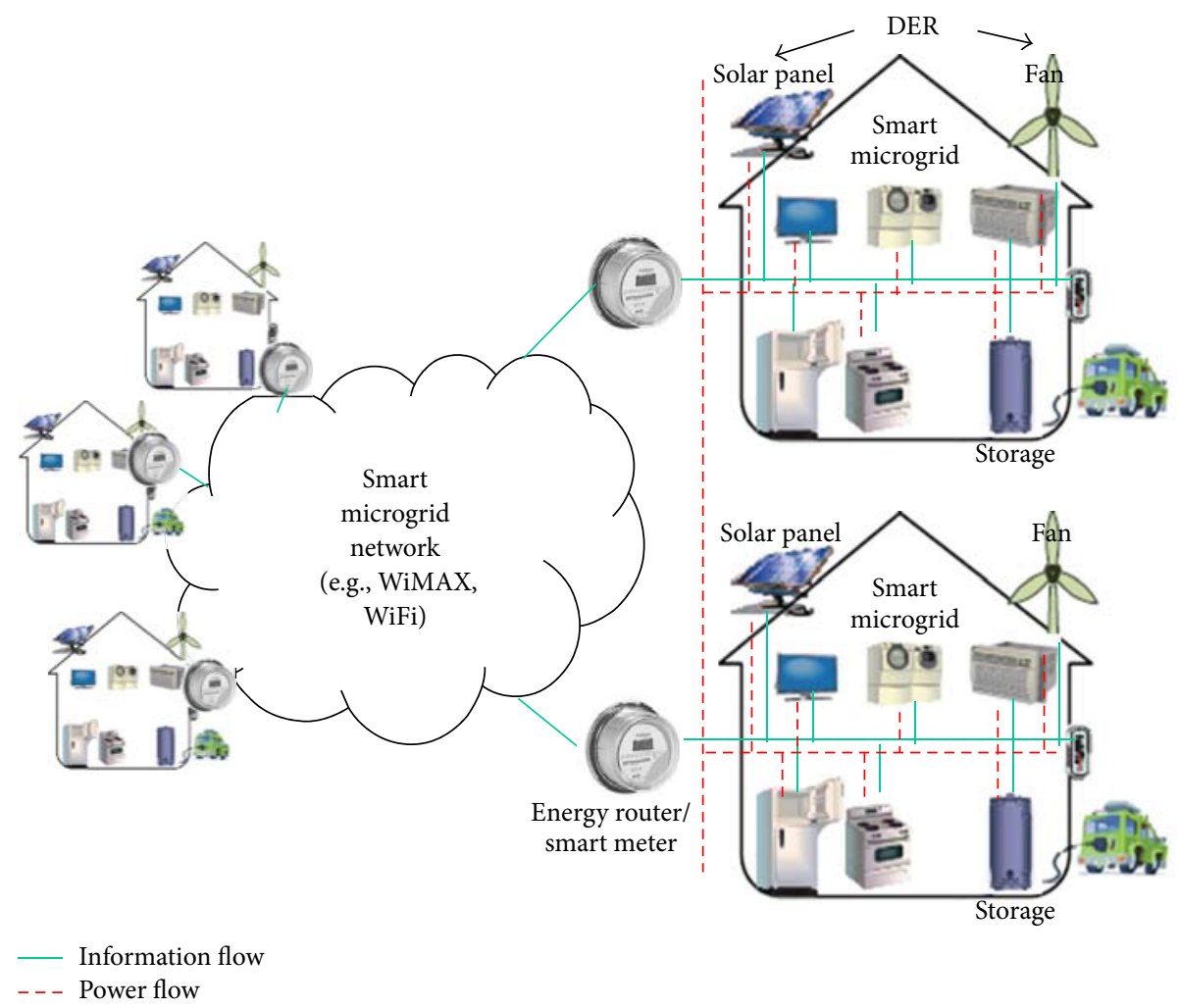

FIGURE 1: Smart microgrid network with renewable energy in smart grid.

network, energy flows from one home to another through the most energy-efficient path on the energy plane of the energy network, which is called energy routing $[3,4]$.

As recent related work, the authors in [4] proposed an energy routing algorithm with distributed agility to avoid failures that demonstrably maximizes the carrying capacity of existing power-line resources. However, this algorithm is impractical because it does not consider an effective transaction mechanism, including a pricing strategy. In this paper, we propose a strategy to determine the desired sale and purchase prices for the surplus and shortage of electricity, respectively, and an optimization scheme [5-10] for finding the most efficient energy route.

The remainder of this paper is organized as follows. Section 2 introduces previous works related to a smart microgrid and energy routing. Section 3 defines the system model addressed in this paper, and Section 4 proposes a gametheory-based energy routing approach for the described problem. Section 5 then describes the simulation results for an energy stock based on the price strategies used in artificial microgrids. Finally, Section 6 provides some concluding remarks regarding this research.

\section{Related Work}

A smart microgrid is similar to a small-scale smart grid and has abundant distributed generators and consumers for medium-/low-voltage electric power within close locations but requires a control mechanism, such as an advanced metering infrastructure (AMI) of a smart grid, to efficiently share the renewable energy generated. Therefore, the generators and consumers are the same as in a home area network (HAN) in a smart grid, and the smart microgrid network (SMGN) connecting them has a similar role as a neighborhood area network (NAN), as shown in Figure 1 [11]. A smart microgrid can benefit from less transmission losses and cable costs because of the close vicinity between the generator and consumers. Moreover, it can decrease the amount of carbon emissions and increase the resilience of the utility grid $[12,13]$.

To share renewable energy efficiently between DERs, energy routing (i.e., setting up an energy-efficient path) has been brought up as a novel feature in an SMGN $[4,14,15]$. In [4], the authors proposed a novel stochastic framework, leveraging distributed storage for alleviating many of the problems of current grids, for example, the difficulty in routing renewable sources owing to their stochastic and often volatile nature. In [15], to maximally utilize the distributed energy resources and minimize the energy transmission overhead, the authors developed distributed energy routing protocols for a smart grid, which can be also applied to a smart microgrid. The authors in [16] proposed a secure energy routing mechanism, and the authors in [17] showed through a simulation that false data injection attacks against distributed energy routing can effectively disrupt the effectiveness of the energy distribution process, thereby generating a significant loss in the energy supply, as well as increases in the energy 
transmission costs and the number of users experiencing power outages. To the best of your knowledge, there are no energy routing mechanisms to consider determining the energy transaction price in pursuit of the maximization of each node's profits.

Game theory is defined as "the study of mathematical models of conflict and cooperation between intelligent rational decision makers" [18]. Thus, game theory has been used in the field of economics and has attracted research interest in the field of communication networking, for example, for the analysis and modeling of routing protocols [19-21]. Game theory is utilized in a general routing decision to enhance the fairness [22] or load balance [23]. While traditional and energy routing are both similar from the viewpoint of the best routing selection, the process factors of the latter approach differ from traditional routing. Therefore, for this paper, we designed an approach based on game theory for energy routing in a smart microgrid.

\section{System Model}

In this section, we present the architecture of an SMGN as a description of the current environment of the energy routing problem. The SMGN used consists of $N$ nodes and $L$ links, such as in a general network used in graph theory. Each microgrid node indicates a home or town with small storage facilities, renewable generators, and power consumers. We consider each microgrid as a player participating in the power exchange games. Let $Q_{p}(k, t)$ and $Q_{c}(k, t)$ represent the total amount of electricity production and consumption by all generators and consumers in the $k$ th microgrid at time period $t$, respectively. The total amount of electricity stored in various storage facilities in node $k$ at the beginning of time period $t$ is represented as $U(k, t)$.

The node has a surplus or shortage of electricity according to the size of $Q_{p}(k, t)$ and $Q_{c}(k, t)$, respectively. If $U(k, t)+$ $Q_{p}(k, t)-Q_{c}(k, t)$ is greater than 0 , node $k$ becomes the supply node in the SMGN at time period $t$; otherwise, node $k$ becomes the demand node. We define $S(k, t)$ as the amount of electricity surplus in node $k$ at time period $t$ and $D(k, t)$ as the amount of electricity shortage, through the following equations:

$$
\begin{aligned}
& S(k, t)=\max \left\{U(k, t)+Q_{p}(k, t)-Q_{c}(k, t), 0\right\}, \\
& D(k, t)=-\min \left\{U(k, t)+Q_{p}(k, t)-Q_{c}(k, t), 0\right\} .
\end{aligned}
$$

At this point, considering the storage capacity of the power storage device, $W(k)$, we can split the amount of surplus of the supply node into two parts: $S_{1}(k, t)$ and $S_{2}(k, t)$. Here, $S_{1}(k, t)$ is the amount of power in excess of the storage capacity among the electricity surpluses. This excess energy cannot be stored, and it is thus advantageous to sell at a price lower than the production cost at the node because it is wasteful to not sell during this period. In addition, $S_{2}(k, t)$ is the amount of power under the storage capacity among the electricity surplus. By not being sold, this energy can be stored at a storage facility during this period and can be used in the next period. Therefore, it is advantageous to choose a pricing strategy that seeks out the greatest profit through such a transaction. We define $S_{1}(k, t)$ and $S_{2}(k, t)$ through the following equations:

$$
\begin{aligned}
& S_{1}(k, t) \\
& =\max \left\{U(k, t)+Q_{p}(k, t)-Q_{c}(k, t)-\delta \cdot W(k), 0\right\}, \\
& S_{2}(k, t) \\
& \quad=\min \left\{U(k, t)+Q_{p}(k, t)-Q_{c}(k, t), \delta \cdot W(k)\right\}, \\
& S(k, t)=S_{1}(k, t)+S_{2}(k, t),
\end{aligned}
$$

where $0 \leq \delta \leq 1$ - the rate of proper charging capacity for the storage device

The amount of electricity demand during a power shortage (defined as $D(k, t)$ above) is the amount of purchased electricity with the highest priority for power use. However, the demand node is favorable for buying and storing a specific amount of electricity when it can purchase energy at a price close to (or lower than) the production cost. Therefore, the demand node may have an extra amount of demand, $D_{2}(k, t)$, and the final power demand, $D(k, t)$, is then updated using $D_{1}(k, t)$, which is the same as $D(k, t)$ defined above, and $D_{2}(k, t)$ through the following equations:

$$
\begin{aligned}
& D_{1}(k, t)=-\min \left\{U(k, t)+Q_{p}(k, t)-Q_{c}(k, t), 0\right\}, \\
& D_{2}(k, t)=\delta \cdot W(k), \\
& D(k, t)=D_{1}(k, t)+D_{2}(k, t) .
\end{aligned}
$$

The link from node $i$ to another node $j$ is labeled as ordered node pair $(i, j)$, which is utilized for power transmissions. Node $i$, with an electricity surplus, sends some of its power, which node $j$, with an electricity shortage, receives; in this case, nodes $i$ and $j$ use link $(i, j)$ for the power transmission. Let $x_{i j}(t)$ be the transmission rate over link $(i, j)$ during time slot $t$. To determine the optimal transmission rate, $x_{i j}^{*}(t)$, the system should choose the price of the electricity sold by each transmitting node and the price of electricity bought by each receiving node.

To do so, we suppose the use of a control center (CC) of the SMGN for the power exchange. A supply node intends to sell its surplus electricity to the demand nodes, and a demand node intends to buy electricity from the supply nodes. The $\mathrm{CC}$ receives information regarding the amount of electricity to be sold or bought from the supply and demand nodes, respectively, and matches the supply node to the demand node based on the amount of electricity to be transmitted. We define the amount of electricity to sell to the demand nodes for supply node $k$ at time period $t$ and the amount of electricity to buy from the supply nodes for demand node $k$ at time period $t$, that is, $Q_{s}(k, t)$ and $Q_{b}(k, t)$, respectively, as follows:

$$
\begin{aligned}
& Q_{s}(k, t)=\sum_{\forall j \neq k} x_{k j}^{*}(t), \\
& Q_{b}(k, t)=\sum_{\forall i \neq k} x_{i k}^{*}(t),
\end{aligned}
$$

where $0 \leq Q_{s}(k, t) \leq S(k, t) ; 0 \leq Q_{b}(k, t) \leq D(k, t)$. 
The amount of electricity stored in node $k$ at the end of time period $t$, that is, $U(k, t+1)$, satisfies the following equality:

$$
U(k, t+1)= \begin{cases}U(k, t)+Q_{p}(k, t)-Q_{c}(k, t)-Q_{s}(k, t), & \text { for supply node } k \\ U(k, t)+Q_{p}(k, t)-Q_{c}(k, t)+Q_{b}(k, t), & \text { for demand node } k .\end{cases}
$$

\section{Game-Theory-Based Energy Routing Approach}

4.1. Problem Definition. Our objective in this research is to find the optimal energy route to meet the electricity demand for all nodes in an SMGN. To achieve this goal, we divide this problem into two subproblems:

(i) The first subproblem is for each selling/buying node to find the proper price to maximize its own profit.

(ii) The second subproblem is for each node to determine the optimal power transmission path according to a potential counterparty and the price chosen by the CC.

In this research, we introduce certain assumptions to determine the proper price for the first subproblem. Each node has the average production expense (normal production expense, $\left.C_{n}(k)\right)$ for the typical power production and the average production expense (additional production expense, $C_{a}(k)$ ) for additional power production. In the remaining cases, each node intends to sell its surplus electricity at over the normal production cost, $C_{n}(k)$, after using the typical amount of power produced, and thus $C_{n}(k)$ is the lower bound of the desired sale price. Similarly, each node does not intend to supplement its electricity shortage at over the additional production cost, $C_{a}(k)$, during a shortage period after using its typical power production, and thus $C_{a}(k)$ is the upper bound of the desired purchase price.

The CC adopts a stock exchange pricing scheme to determine the energy transaction price during time period $t$. Thus, each supply and demand node provides its amount of electricity to sell and buy along with the desired sale and purchase price, respectively, to the CC. The CC matches the amount of electricity to be sold by the supply nodes in ascending order of their desired sale price with the amount of electricity to purchase by the demand nodes in descending order of their desired purchase price. The CC then determines the energy transaction price of the matching point, which is defined as $P(t)$.

4.2. Pricing Strategy and Transportation Problem. In this decision scheme for determining the trading price, the lower the desired sale price suggested by the supply node is, the more likely the supply node will be able to sell all of its surplus electricity, but at a decrease in profit. Meanwhile, the higher the desired purchase price suggested by the demand node is, the more likely the demand node will be able to purchase all of its required electricity needs, but at an increase in the purchasing cost. Each node as game player has the desired sale and purchase prices as selectable strategies. If there is only one supply node and one demand node, payoff table for each selectable strategy is shown in Table 1. In the case that the desired sale price of supply node is less than or equal to the desired purchase price of demand node, energy transaction price is determined with the average of those two prices. Thus, the positive gain of supply node and the negative gain of demand node are equal. In the opposite case, because transaction is not conducted, the gain of supply node is 0 and the negative gain becomes $M$ as electricity shortage cost of demand node. The optimal strategy of supply nodes $i^{*}=\operatorname{argmax}_{i}\left\{p_{i j^{*}}^{s} \mid \operatorname{argmin}_{j}\left(p_{i j}^{d}\right)\right\}$ becomes the biggest price among possible strategies, and the optimal strategy of demand nodes $j^{*}=\operatorname{argmin}_{j}\left\{p_{i^{*} j}^{d} \mid \operatorname{argmax}_{i}\left(p_{i j}^{s}\right)\right\}$ becomes the smallest price among possible strategies. Therefore, the stable point of such transaction game does not exist.

Therefore, in this research, we designed three strategies for the supply and demand nodes to determine the desired price using the trading price $P(t-1)$ for the preceding time period $t-1$. Here, $P_{s}(k, t)$ denotes the desired sale price of supply node $k$ at time $t$. Because we divide the amount of surplus electricity of the supply node into the amount of power in excess of storage capacity $S_{1}(k, t)$ and the amount of power under storage capacity $S_{2}(k, t)$, we define these two types of desired sales prices as $P_{s_{1}}(k, t)$ and $P_{s_{2}}(k, t)$, respectively. The amount of power in excess of the storage capacity can be sold at a lower price than the production cost, whereas, for the amount of power below the storage capacity, the desired sales price that compensates for the production costs should be chosen. Here, $P_{b}(k, t)$ denotes the desired purchase price of demand node $k$ at time $t$. Similarly, we define the two types of desired purchase prices for the electricity shortage of the demand node as $P_{b_{1}}(k, t)$ and $P_{b_{2}}(k, t)$, where $P_{b_{1}}(k, t)$ is the price for the electricity demand for power shortage $D_{1}(k, t)$ and $P_{b_{2}}(k, t)$ is the price for the extra demanded electricity $D_{2}(k, t)$, which is stored for use during the next period. For $D_{1}(k, t)$, that is, the power to be bought, the price $P_{b_{1}}(k, t)$ will be close to the cost of the excess production. The price $P_{b_{2}}(k, t)$ should be set lower than the production cost for the extra demanded power, $D_{2}(k, t)$.

We suggest three strategies for determining the desired prices of the supply and demand nodes, considering each of the above points.

Strategy 1 (optimistic strategy). It is a decision strategy for the desired trading price, $P_{s}(k, t)$ and $P_{b}(k, t)$, to maximize the profit, rather than the possibility of the sale/purchase 
TABLE 1: Payoff table $\left(p_{i j}^{s},-p_{i j}^{d}\right)$ for transaction game.

\begin{tabular}{lccccc}
\hline Seller & \multicolumn{5}{c}{ Buyer } \\
\hline$\vdots$ & 4 & 5 & 6 & 7 & \\
\hline & & & & \\
5 & $(4,-4)$ & $(4.5,-4.5)$ & $(5,-5)$ & $(5.5,-5.5)$ & $(6,-6)$ \\
5 & $(0,-M)$ & $(5,-5)$ & $(5.5,-5.5)$ & $(6,-6)$ & $(6.5,-6.5)$ \\
6 & $(0,-M)$ & $(0,-M)$ & $(6,-6)$ & $(6.5,-6.5)$ & $(7,-7)$ \\
7 & $(0,-M)$ & $(0,-M)$ & $(0,-M)$ & $(7,-7)$ & $(7.5,-7.5)$ \\
8 & $(0,-M)$ & $(0,-M)$ & $(0,-M)$ & $(0,-M)$ & $(8,-8)$ \\
$\vdots$ & & & & \\
\hline
\end{tabular}

being predicted to similarly determine price $P(t)$ based on the trading price $P(t-1)$ during the preceding time period

$$
\begin{aligned}
& P_{s_{1}}(k, t)=C_{n}(k), \\
& P_{s_{2}}(k, t)=\max \left\{P(t-1), \frac{C_{n}(k)+C_{a}(k)}{2}\right\}, \\
& P_{b_{1}}(k, t)=\min \left\{P(t-1), \frac{C_{n}(k)+C_{a}(k)}{2}\right\}, \\
& P_{b_{2}}(k, t)=\frac{3 \cdot C_{n}(k)-C_{a}(k)}{2} .
\end{aligned}
$$

Strategy 2 (medium strategy). It is a decision strategy for the desired trading price, $P_{s}(k, t)$ and $P_{b}(k, t)$, to pursue the maximization of both the possibility of a sale/purchase and profits from trading:

$$
\begin{aligned}
& P_{s_{1}}(k, t)=\frac{5 \cdot C_{n}(k)-C_{a}(k)}{4}, \\
& P_{s_{2}}(k, t) \\
& \quad=\max \left\{\frac{C_{n}(k)+P(t-1)}{2}, \frac{3 \cdot C_{n}(k)+C_{a}(k)}{4}\right\}, \\
& P_{b_{1}}(k, t) \\
& \quad=\min \left\{\frac{P(t-1)+C_{a}(k)}{2}, \frac{C_{n}(k)+3 \cdot C_{a}(k)}{4}\right\}, \\
& P_{b_{2}}(k, t)=\frac{5 \cdot C_{n}(k)-C_{a}(k)}{4} .
\end{aligned}
$$

Strategy 3 (pessimistic strategy). It is a decision strategy for the desired trading price, $P_{s}(k, t)$ and $P_{b}(k, t)$, to maximize the possibility of a sale or purchase, rather than a profit, when predicting a quite different determination of price $P(t)$ from trading price $P(t-1)$ based on the preceding time period:

$$
\begin{aligned}
& P_{s_{1}}(k, t)=\frac{3 \cdot C_{n}(k)-C_{a}(k)}{2}, \\
& P_{s_{2}}(k, t)=C_{n}(k), \\
& P_{b_{1}}(k, t)=C_{a}(k), \\
& P_{b_{2}}(k, t)=C_{n}(k) .
\end{aligned}
$$

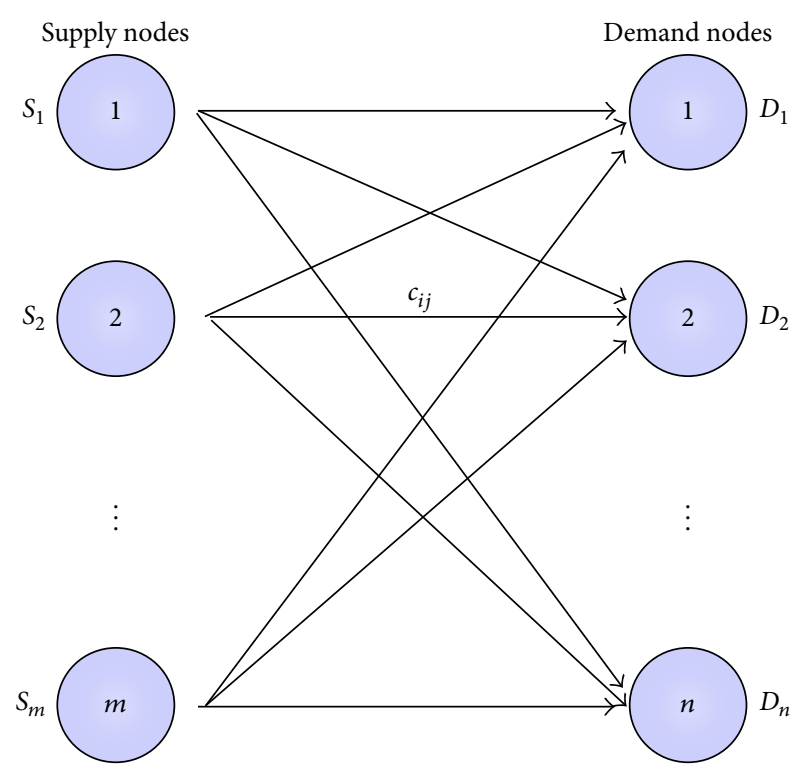

FIGURE 2: Architecture of traditional transportation problem.

After solving the first subproblem, the CC obtains the transaction price $P(t)$ at time period $t$, the amount of electricity sold by each supply node, and the amount of electricity purchased by each demand node. Utilizing the results of the first subproblem, the CC then chooses the proper path for transferring the power as the second subproblem. The second subproblem has an architecture exactly the same as that of a traditional transportation problem (see Figure 2) because it is a problem of finding the optimum power transmission path between the chosen supply and demand nodes while satisfying the amount of electricity demanded by the demand node. Moreover, this is considered a balanced transportation problem because the total amount of electricity sold, $Q_{s}(k, t)$, as determined by the CC, is equal to the total amount of electricity purchased, $Q_{b}(k, t)$, which is also chosen by the CC.

4.3. Energy Routing Optimization. The decision problem of the trading price for the $\mathrm{CC}$, which takes on the role of the power exchanger, corresponds to a type of classic multiplayer competitive game. Therefore, different strategies are required 
based on the situation, which in our problem is determined according to the total amounts of the electricity surplus and shortage. The optimal strategy for the supply and demand nodes, based on the situation, is as follows.

Situation 1 (excess supply). It is a case in which the total amount of surplus electricity in the SMGN exceeds the total amount of electricity shortage:

$$
\sum_{k \in \text { Supply Nodes }} S(k, t)>\sum_{k \in \text { Demand Nodes }} D(k, t) .
$$

In this situation, the probability of a sale by a supply node decreases significantly, and thus Strategy 3 (pessimistic) is suitable for a supply node. In contrast, the purchase probability of a demand node increases greatly, and thus a demand node has an advantage when selecting Strategy 1 (optimistic) to increase its profits.

Situation 2 (equilibrium). It is a case in which the total amount of surplus electricity in the SMGN is the same as the total amount of electricity shortage:

$$
\sum_{k \in \text { Supply Nodes }} S(k, t)=\sum_{k \in \text { Demand Nodes }} D(k, t) .
$$

In this situation, the sale probability of the supply nodes is at a similar level as the purchase probability of the demand nodes. Thus, Strategy 2 (medium) is a good choice for both supply and demand nodes.

Situation 3 (excess demand). It is a case in which the total amount of surplus electricity in the SMGN is lower than the total amount of electricity shortage:

$$
\sum_{k \in \text { Supply Nodes }} S(k, t)<\sum_{k \in \text { Demand Nodes }} D(k, t) .
$$

Under excess demand, Strategy 1 (optimistic) is a good choice for the supply nodes because this situation heightens the sale probability considerably. Moreover, Strategy 3 (pessimistic), forgoing its profit, is proper for a demand node because the purchase probability is very low.

At the beginning of time period $t$, each supply node submits its chosen sale price, $P_{s}(k, t)$, and the amount of electricity for sale, $S(k, t)$, according to its selected strategy, to the CC. Each demand node also submits its chosen purchase price, $P_{b}(k, t)$ and the amount of electricity for purchase, $D(k, t)$, based on its selected strategy, to the CC. The CC then determines the trading amount for each demand node by utilizing the matching algorithm in order of the desired transaction price.

The energy routing problem used for this decision can be used to find the optimum power transmission path, $x_{i j}^{*}(t)$, utilizing the Hungarian algorithm (developed by Kuhn in 1955), which is a well-known solving method for the transportation problem [24]. Moreover, it was proved that the Hungarian algorithm has a polynomial time complexity because it uses only a matrix operation.

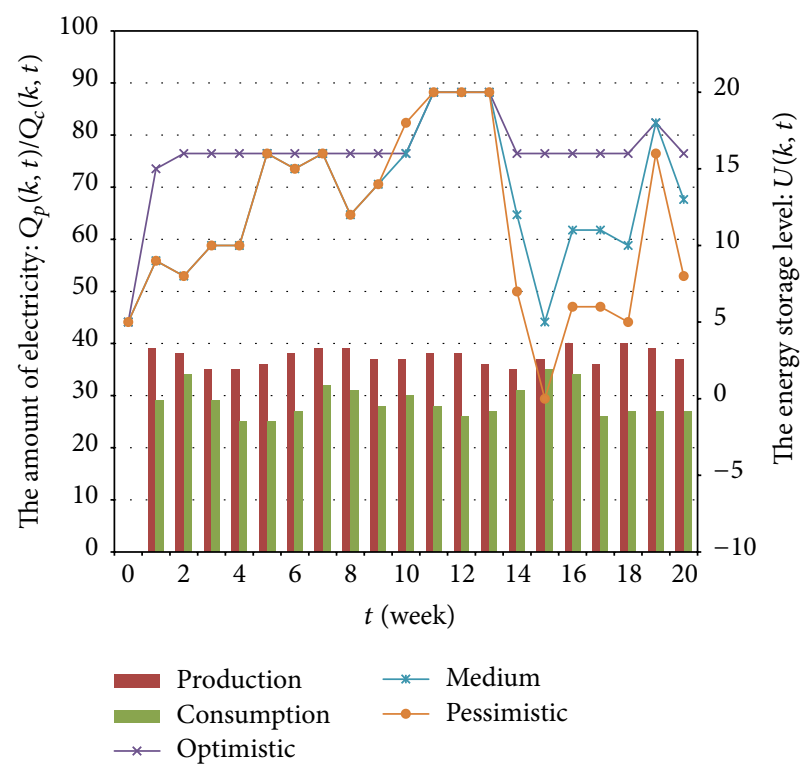

FIGURE 3: Trend of energy storage level at microgrid NC.

\section{Experimental Results}

We assume three locations, NC, SC, and GA, for the microgrids used in the simulation and set the different characteristics for each. NC has more electricity production than consumption, the electricity consumption of SC exceeds its production, and GA has similar amounts of energy production and consumption. We identify the characteristics of the three strategies (i.e., optimistic, medium, and pessimistic) by applying the strategies to these three microgrid locations.

For the results of NC with an excess electricity supply (see Figure 3), the microgrid does not sell its accumulated energy surplus and, under the optimistic strategy, has a consistently high level of electricity stock. Thus, we can confirm that a waste of energy owing to an excessive storage capacity exists. In the usage case for the medium strategy, NC maintains a low amount of power stock through the proper sale of its excess electricity, which is similar to the results of the pessimistic strategy. However, the use of the pessimistic strategy decreases NC's profits through offering a lower selling price than in the medium strategy.

In contrast, for SC (i.e., a microgrid whose power consumption exceeds its production), using the optimistic strategy does not solve the problem of a lack of power through purchasing, and the power shortage is maintained (see Figure 4). Under the medium strategy, SC nearly maintains a state without a power shortage or excess through a sufficient purchasing of power, whereas, under the pessimistic strategy, SC secures some excess electricity through an aggressive purchase.

For the results of the GA microgrid, which has similar levels of electricity production and consumption (see Figure 5), the optimistic strategy pursues maximized profits through electricity transactions. Thus, energy depletion is shown during periods of high electricity consumption, and a high level of electricity stockpiling is shown for periods with 


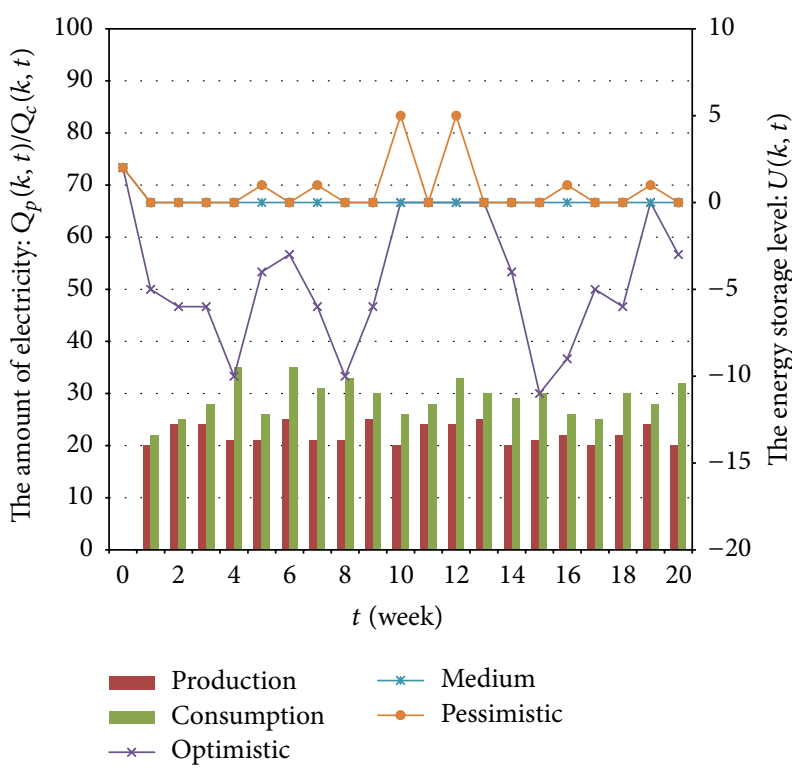

FIGURE 4: Trend of energy storage level at microgrid SC.

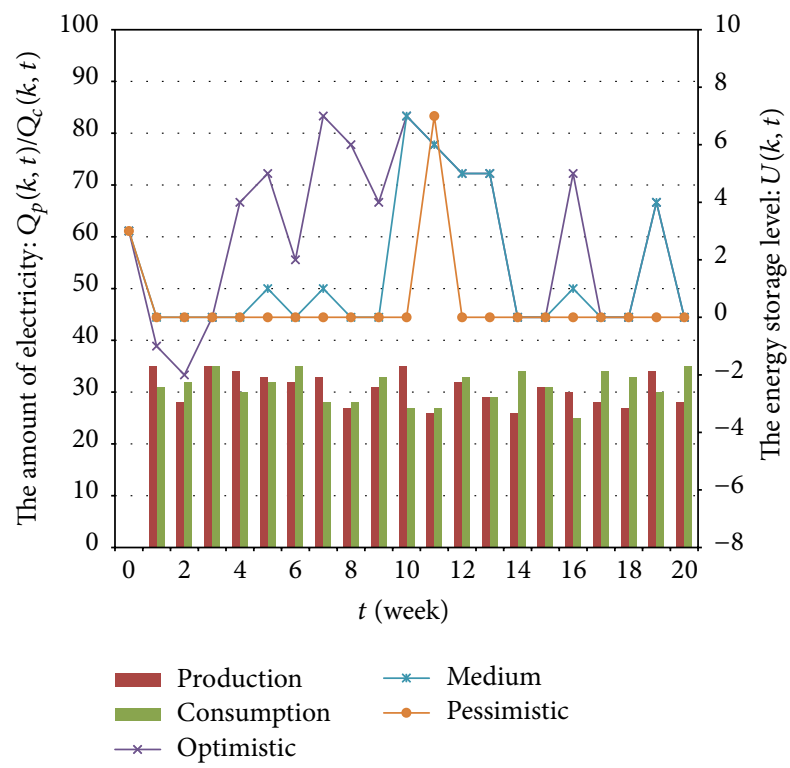

FIgURE 5: Trend of energy storage level at microgrid GA.

high electricity production. Using the medium strategy, GA maintains the proper level of stockpiling through its purchasing of electricity during periods of high power consumption and through its selling of electricity during periods of high power production. Under the pessimistic strategy, GA's power reserves are nearly depleted. Thus, GA appears to have the optimum status but suffers from reduced profits from energy trading owing to its low sales price.

To verify the results based on these three strategies from another perspective, we demonstrated the variations in the transaction price determined by the seller and buyer, as shown in Figure 6. In this figure, a lack of price points

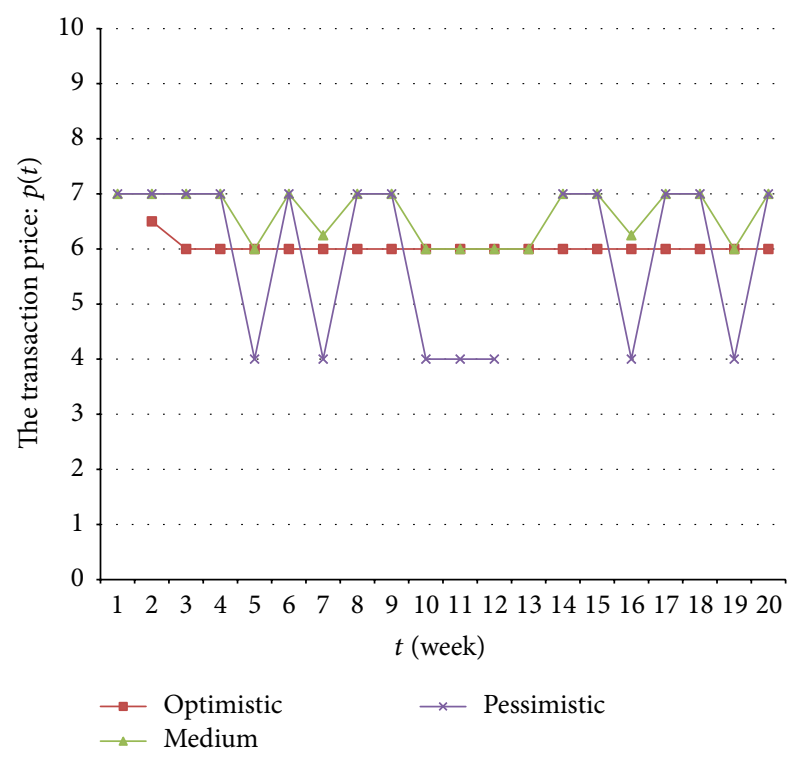

FIGURE 6: Trend of transaction price under different pricing strategies.

TABLE 2: Total profit of each node by electricity transaction.

\begin{tabular}{lccc}
\hline Node & $\begin{array}{c}\text { Strategy } \\
\text { Strategy 1 } \\
\text { (Optimistic) }\end{array}$ & $\begin{array}{c}\text { Strategy 2 } \\
\text { (Medium) }\end{array}$ & $\begin{array}{c}\text { Strategy 3 } \\
\text { (Pessimistic) }\end{array}$ \\
\hline NC & 768 & 908.75 & 784 \\
SC & $-13 M-738$ & -863 & -681 \\
GA & -30 & -45.75 & -103 \\
\hline
\end{tabular}

indicates that the trading conditions of the seller and buyer were not met and no business transactions took place.

Initially, under the optimistic strategy, the deals do not work well and are thus sustained at the average transaction price. In contrast, under the pessimistic strategy, the seller and buyer trade actively (i.e., with the price strategy minimizing their profits) according to the variations in supply and demand, and thus the transaction price changes rapidly. The medium strategy shows moderate variations in the transaction price, which hovers near the average price.

According to the above electricity transaction prices, the summations of gain obtained at each node are shown in Table 2.

\section{Conclusions}

In a smart microgrid network (SMGN), which has been predicted to be one of the core components of a future power grid, the optimal power transmission problem is an important factor. In this paper, we proposed an optimal price decision strategy according to the particular situation based on a power exchange. The power exchange determines the proper counterparty by utilizing an affordable amount for surplus electricity, the desirable selling price for such surplus, the amount of electricity demand, and the desired purchase price during a shortage period. We also modeled 
an energy routing approach satisfying the amount of electricity supply/demand, as the same problem, and suggested a potential solution. The proposed strategy and energy routing methodology will contribute to the introduction of a smart power grid, increasing the possibility of its utilization.

As future work, we plan to compare a combined strategy that applies a probabilistically selected pricing strategy to the market using the pure strategies proposed in this paper and evaluate its performance enhancement. Moreover, we plan to evaluate our proposed scheme using real network data to verify the effectiveness and efficiency of a real-world SMGN.

\section{Conflict of Interests}

The authors declare that there is no conflict of interests regarding the publication of this paper.

\section{Acknowledgment}

This work was supported by Kyonggi University Research Grant 2013.

\section{References}

[1] Smart Micro Grid Campus Project Belecke, 2014, https://www .aegps.com/en/applications/storage-distribution/smart-microgrid/.

[2] M. Yoon, Y.-K. Kim, and J.-W. Chang, "An energy-efficient routing protocol using message success rate in wireless sensor networks," Journal of Convergence, vol. 4, no. 1, pp. 15-22, 2013.

[3] F. Bouhafs, M. Merabti, and A. Hardy, "A communication architecture for power routing in the smart grid," in Proceedings of the 1st International Conference and Exhibition on the Applications of Information Technology to Renewable Energy Processes and Systems (IT-DREPS '13), pp. 123-126, Amman, Jordan, May 2013.

[4] M. Baghaie, S. Moeller, and B. Krishnamachari, "Energy routing on the future grid: a stochastic network optimization approach," in Proceedings of the International Conference on Power System Technology (POWERCON '10), pp. 1-8, IEEE, Hangzhou, China, October 2010.

[5] T.-J. Kim, B.-G. Park, and K.-S. Jang, "Efficient block mode determination algorithm using adaptive search direction information for scalable video coding (SVC)," Journal of Convergence, vol. 5, pp. 23-29, 2014.

[6] M. M. Weng, T. K. Shih, and J. C. Hung, "A personal tutoring mechanism based on the cloud environment," Journal of Convergence, vol. 4, no. 3, pp. 37-44, 2013.

[7] M. Juneja and P. S. Sandhu, "A new approach for information security using an improved steganography technique," Journal of Information Processing Systems, vol. 9, no. 3, pp. 405-424, 2013.

[8] M. Gohar and S.-J. Koh, "A network-based handover scheme in HIP-based mobile networks," Journal of Information Processing Systems, vol. 9, no. 4, pp. 651-659, 2013.

[9] M. I. Malkawi, "The art of software systems development: Reliability, Availability, Maintainability, Performance (RAMP)," Human-Centric Computing and Information Sciences, vol. 3, no. 1, pp. 1-22, 2013.

[10] H. T. T. Binh and S. H. Ngo, "All capacities modular cost survivable network design problem using genetic algorithm with completely connection encoding," Human-Centric Computing and Information Sciences, vol. 4, pp. 1-13, 2014.

[11] M. Kim, "Toward smart microgrid with renewable energy: an overview of network design, security, and standards," in Computational Science and Its Applications-ICCSA 2013, vol. 7971 of Lecture Notes in Computer Science, pp. 142-156, Springer, Berlin, Germany, 2013.

[12] M. Erol-Kantarci, B. Kantarci, and H. T. Mouftah, "Reliable overlay topology design for the smart microgrid network," IEEE Network, vol. 25, no. 5, pp. 38-43, 2011.

[13] S. Alwala, A. Feliachi, and M. A. Choudhry, "Multi agent system based fault location and isolation in a smart microgrid system," in Proceedings of the IEEE PES Innovative Smart Grid Technologies (ISGT '12), pp. 1-4, IEEE, Washington, DC, USA, January 2012.

[14] Y. M. Kwon, J. S. Kim, M. Y. Chung, H. Choo, T. J. Lee, and M. Kim, "State of the art 3GPP M2M communications toward smart grid," KSII Transactions on Internet and Information Systems, vol. 6, no. 2, pp. 468-479, 2012.

[15] J. Lin, W. Yu, D. Griffith, X. Yang, G. Xu, and C. Lu, "On distributed energy routing protocols in the smart grid," in Software Engineering, Artificial Intelligence, Networking and Parallel/Distributed Computing, vol. 492 of Studies in Computational Intelligence, pp. 143-159, Springer, Basel, Switzerland, 2013.

[16] T. Zhu, S. Xiao, Y. Ping, D. Towsley, and W. Gong, "A secure energy routing mechanism for sharing renewable energy in smart microgrid," in Proceedings of the IEEE International Conference on Smart Grid Communications (SmartGridComm '11), pp. 143-148, IEEE, Brussels, Belgium, October 2011.

[17] J. Lin, W. Yu, X. Yang, G. Xu, and W. Zhao, "On false data injection attacks against distributed energy routing in smart grid," in Proceedings of the IEEE/ACM 3rd International Conference on Cyber-Physical Systems (ICCPS '12), pp. 183-192, Beijing, China, April 2012.

[18] R. B. Myerson, Game Theory: Analysis of Conflict, Harvard University Press, 1991.

[19] S. P. Algur and N. P. Kumar, "Novel user centric, game theory based bandwidth allocation mechanism in WiMAX," HumanCentric Computing and Information Sciences, vol. 3, pp. 1-20, 2013.

[20] S.-K. Bae, "Power consumption analysis of prominent time synchronization protocols for wireless sensor networks," Journal of Information Processing Systems, vol. 10, no. 2, pp. 300-313, 2014.

[21] F.-N. Pavlidou and G. Koltsidas, "Game theory for routing modeling in communication networks-a survey," Journal of Communications and Networks, vol. 10, no. 3, pp. 268-286, 2008.

[22] F. Ahourai, M. Tabandeh, M. Jahed, and B. Afsari, "A fair routing algorithm for wireless mesh networks based on game theory," in Proceedings of the 8th International Conference on Networks (ICN '09), pp. 144-149, IEEE, Cancun, Mexico, March 2009.

[23] H. Tian, F. Jiang, and W. Cheng, "A game theory based loadbalancing routing with cooperation stimulation for wireless ad hoc networks," in Proceedings of the 11th IEEE International Conference on High Performance Computing and Communications (HPCC '09), pp. 266-272, IEEE, Seoul, South Korea, June 2009.

[24] H. W. Kuhn, "The Hungarian method for the assignment problem," Naval Research Logistics Quarterly, vol. 2, pp. 83-97, 1955. 


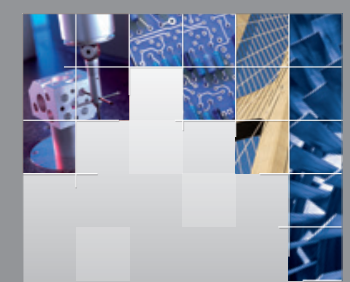

\section{Enfincering}
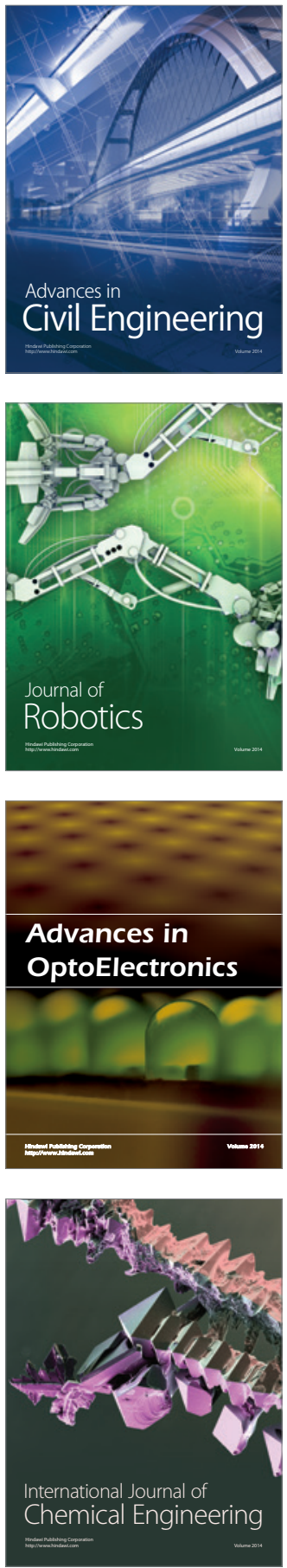

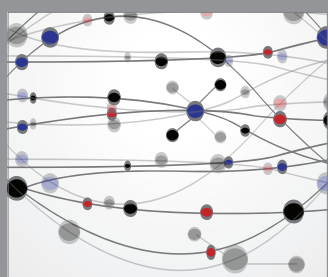

The Scientific World Journal

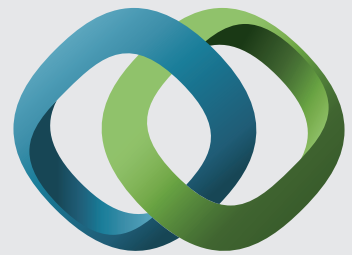

\section{Hindawi}

Submit your manuscripts at

http://www.hindawi.com
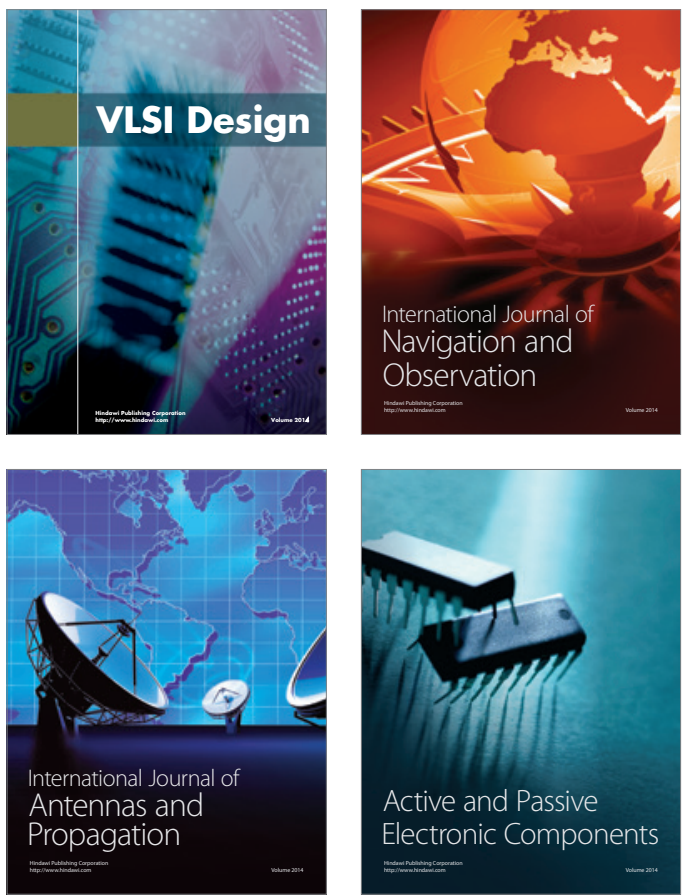
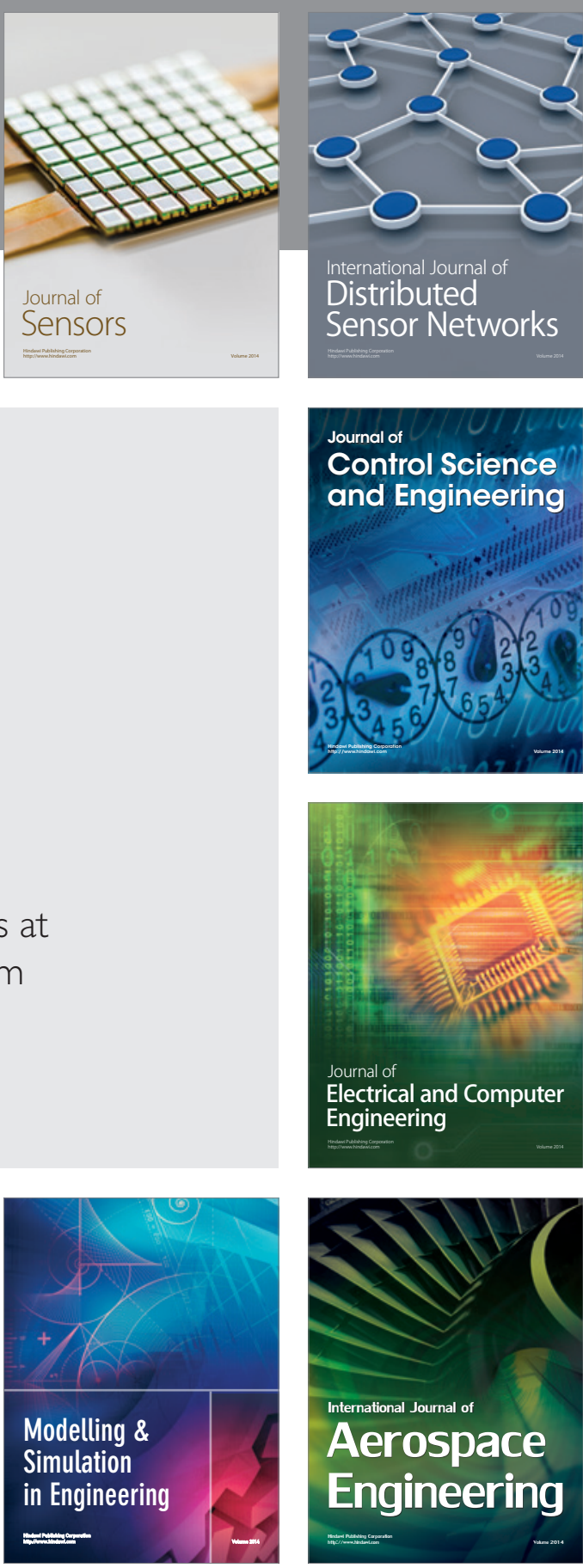

International Journal of

Distributed

Sensor Networks

Journal of

Control Science

and Engineering
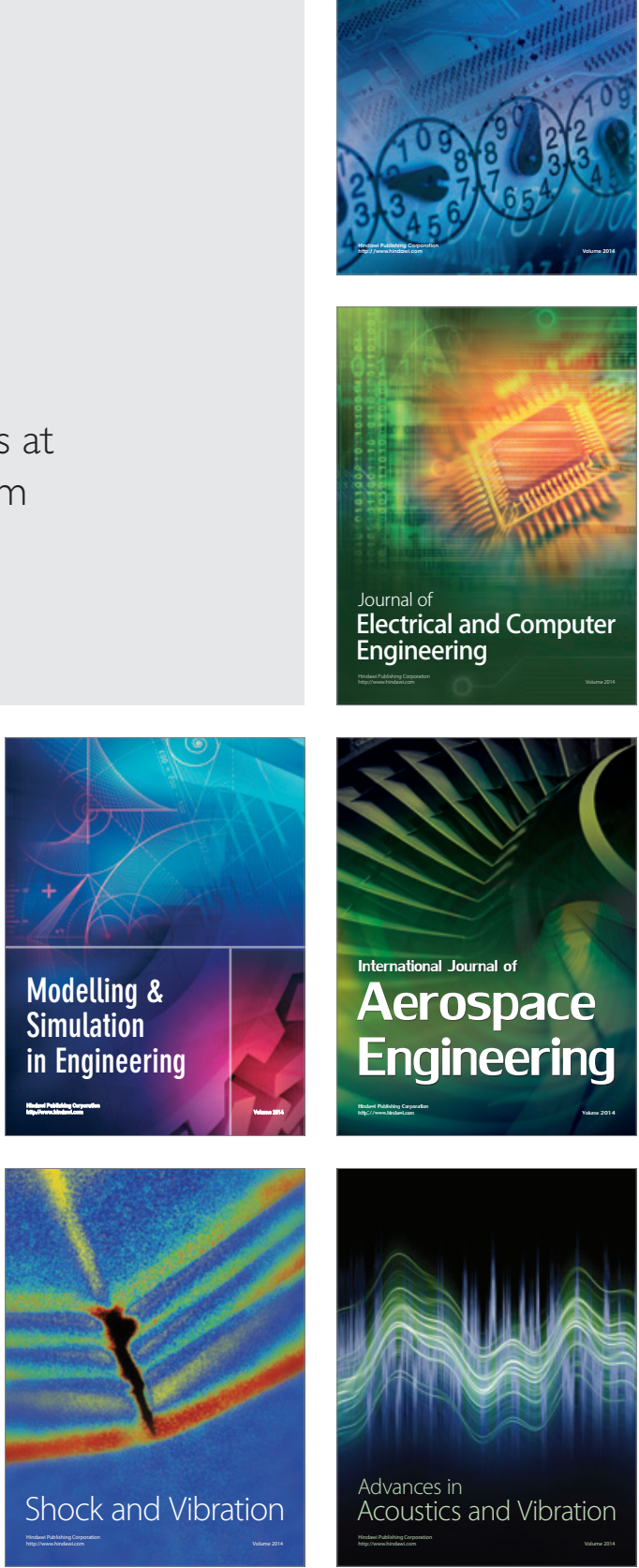